VOL. $5(1971), 227-238$.

\title{
On some mean value theorems of the differential calculus
}

\author{
J.B. Diaz and R. Výborný
}

\begin{abstract}
A general mean value theorem, for real valued functions, is proved. This mean value theorem contains, as a special case, the result that for any, suitably restricted, function $f$ defined on $[a, b]$, there always exists a number $c$ in $(a, b)$ such that $f(c)-f(a)=f^{\prime}(c)(c-a)$. A partial converse of the general mean value theorem is given. A similar generalized mean value theorem, for vector valued functions, is also established.
\end{abstract}

\section{Introduction}

Flett's mean value theorem [6], which has attracted some attention (see, for example the book by Boas [2]), was generalized by Lakshminarasimhan [7], Trahan [9] and Reich [8]. Flett's Theorem reads: If $f(x)$ is a differentiable real valued function on $[a, b]$, and $f^{\prime}(a)=f^{\prime}(b)$, then there exists a number $c$ in $(a, b)$ such that $f(c)-f(a)=f^{\prime}(c)(c-a)$. In this note, Flett's Theorem is generalized further; this generalization brings out more clearly the geometrical fact behind Flett's Theorem. Expressed in intuitive geometrical language, Lagrange's mean value theorem says that, given a smooth plane curve $\widehat{A B}$ joining two points $A$ and $B$, there always is a point $C$, interior to the curve $\widehat{A B}$, such that the tangent to the curve at $C$ is parallel to the chord $\overline{A B}$; whereas the present generalization of Flett's Theorem states that, if the curve intersects the chord $\overline{A B}$, then there is a point $D$, interior to the curve $\widehat{A B}$, such that the straight line $A D$ is

Received 15 April 1971. The second author gratefully acknowledges support from the Albert Einstein Chair of Science at Rensselaer Polytechnic Institute. 
tangent to the curve at $D$. A partial converse of this theorem is also given. Besides this, a theorem for vector valued functions is also proved.

\section{Real valued functions}

Let $f$ and $g$ be real valued functions, defined on a finite closed interval $[a, b]$, where $a<b$. The set of all points $(g(x), f(x))$, for $x \in[a, b]$, will be called the graph of the couple $(g, f) ;$ and, in the special case when $g(x)=x$, it will be simply called the graph of the function $f$.

For convenience, the following terminology will be adhered to: The groph of the couple $(g, f)$ is said to intersect its chord (internally) provided that there exists a number $\bar{x} \in(a, b)$ such that

$$
[f(\bar{x})-f(a)][g(b)-g(a)]=[g(\bar{x})-g(a)][f(b)-f(a)] .
$$

The graph of the couple $(g, f)$ is said to intersect its chord in the extended sense, if either there is a number $\bar{x} \in(a, b)$ such that (1) holds, or else $g(b) \neq g(a), \lim _{x \rightarrow a^{+}} \frac{f(x)-f(a)}{g(x)-g(a)}$ exists, and

$$
\lim _{x \rightarrow a^{+}} \frac{f(x)-f(a)}{g(x)-g(a)}=\frac{f(b)-f(a)}{g(b)-g(a)}
$$

THEOREM 1. Let the functions $f$ and $g$ satisfy the following conditions:

(i) $f$ and $g$ are continuous on $[a, b]$,

(ii) $g(x) \neq g(a)$ for $a<x \leq b$,

(iii) the groph of the couple $(g, f)$ intersects its chord in the extended sense.

Then there exists a number $c \in(a, b)$, and two positive numbers, $\delta_{1}, \delta_{2}$, such that: either both inequalities

$$
[f(c)-f(a)][g(c)-g(c-h)] \leq[g(c)-g(a)][f(c)-f(c-h)],
$$

and

$$
[f(c+k)-f(c)][g(c)-g(a)] \leq[g(c+k)-g(c)][f(c)-f(a)]
$$

hold for $0<h \leq \delta_{1}$, and $0<k \leq \delta_{2}$; or both inequalities (3), (4) are 
valid, with the inequality signs reversed, for $0<h \leq \delta_{1}$ and $0<k \leq \delta_{2}$.

Proof. Define the auxiliary function $Q(x)=\frac{f(x)-f(a)}{g(x)-g(a)}$ for $x>a$, and, if (2) holds, define $Q$ also at $x=a$ by the equation $Q(x)=\frac{f(b)-f(a)}{g(b)-g(a)}$. No matter whether (1) or (2) holds, there is a number $\bar{x}$, with $a \leq \bar{x}<b$, such that $Q(\bar{x})=Q(b)$, and the function $Q$ is continuous on $[\bar{x}, b]$. Consequently, the function $Q$ attains either its maximum or its minimum, over $[\bar{x}, b]$, at a number $c$, with $\bar{x}<c<b$. Since the conclusion of the theorem is not affected if the function $f$ is replaced by the function $-f$, it can be supposed that $Q$ attains a maximum at $c$. Then, one has

$$
Q(c-h) \leq Q(c),
$$

for $0<h \leq c-\bar{x}=\delta_{1}$, and

$$
Q(c+k) \leq Q(c),
$$

for $0<k \leq b-c=\delta_{2}$. The inequality (5) means that

$$
\frac{f(c-h)-f(a)}{g(c-h)-g(a)} \leq \frac{f(c)-f(a)}{g(c)-g(a)} \text {. }
$$

Since $g(x) \neq g(a)$, for $a<x \leq b$, and $g$ is continuous, one has that either $g(x)>g(a)$ for $a<x \leq b$, or $g(x)<g(a)$ for $a<x \leq b$. Therefore, the product $[g(c)-g(a)][g(c-h)-g(a)]>0$, and hence

$$
[f(c-h)-f(a)][g(c)-g(a)] \leq[f(c)-f(a)][g(c-h)-g(a)] .
$$

Adding $[f(a)-f(c)][g(c)-g(a)]$ to both sides of the last inequality, one obtains (3). Using the inequality (6) one arrives, in a similar way, at the inequality (4).

REMARK I. If alternative (2) holds, that is $\lim _{x \rightarrow a^{+}} \frac{f(x)-f(a)}{g(x)-g(a)}=\frac{f(b)-f(a)}{g(b)-g(a)}$, then the numbers $\delta_{1}$ and $\delta_{2}$ can be taken to be $c-a$ and $b-c$, respectively.

REMARK 2. Assuming, further, that $f$ is differentiable in $(a, b)$, and choosing $g(x)=x$, it follows from (3) and (4), by passing to the limit as $h \rightarrow 0+$ and $k \rightarrow 0+$, respectively, that 


$$
\begin{aligned}
& f(c)-f(a) \leq f^{\prime}(c)(a-a), \\
& f^{\prime}(c)(c-a) \leq f(c)-f(a),
\end{aligned}
$$

which implies

$$
f(c)-f(a)=f^{\prime}(c)(c-a) \text {. }
$$

This is precisely the conclusion of Flett's Theorem, but obtained here under a weaker hypothesis.

REMARK 3. Without assuming that $f$ is differentiable, but still choosing $g(x)=x$, one obtains from the conclusion of Theorem 1 that, either

$$
\frac{f(c+k)-f(c)}{k} \leq \frac{f(c)-f(a)}{c-a} \leq \frac{f(c)-f(c-h)}{h}
$$

or the reverse inequalities hold, for $0<h \leq \delta_{1}$ and $0<k \leq \delta_{2}$. Passing to the limit as $k \rightarrow 0+, h \rightarrow 0+$, and employing the usurl notation for Dini derivates, one obtains that, either

$$
D^{+} f(c) \leq \frac{f(c)-f(a)}{c-a} \leq D_{-} f(c)
$$

or

$$
D^{-} f(c) \leq \frac{f(c)-f(a)}{c-a} \leq D_{+} f(c)
$$

This is the conclusion of Theorem 1 in [8], except that, in [8], c could conceivably be $b$, which is excluded here. It should also be mentioned that the hypothesis

$$
\left[f^{\prime}(b)-\frac{f(b)-f(a)}{b-a}\right]\left[f^{\prime}(a)-\frac{f(b)-f(a)}{b-a}\right] \geq 0
$$

in Theorem 1 of [8] can be shown to imply hypothesis (iii), for $g(x)=x$, in the present Theorem 1 , except in the trivial case when $f^{\prime}(b)=\frac{f(b)-f(a)}{b-a}$.

The usual Lagrange's mean value theorem reads: if $f$ is a real valued function, continuous on $[a, b]$, and differentiable in $(a, b)$, then there exists a number $c \in(a, b)$ such that $f(b)-f(a)=f^{\prime}(c)(b-a)$. A generalization of Lagrange's mean value theorem, concerning the Dini derivates, appears in the work of W.H. and 
Grace Chisholm Young $[10, \mathrm{p} .10]$, which states that, if $f$ is a real valued function continuous on $[a, b]$, then there exists a number $c$, with $a<c<b$, such that either

$$
D^{+} f(c) \leq \frac{f(b)-f(a)}{b-a} \leq D_{-} f(c)
$$

or

$$
D^{-} f(c) \leq \frac{f(b)-f(a)}{b-a} \leq D_{+} f(c) \text {. }
$$

A further generalization can be given as follows [3, p. 115]: If $f$ is a real valued function continuous on $[a, b]$, then there exists a number $c$ such that either

$$
\frac{f(c+k)-f(c)}{k} \leq \frac{f(b)-f(a)}{b-a} \leq \frac{f(c)-f(c-h)}{h}
$$

hold for all positive $h$ and $k$ such that $c+k \in(a, b), c-h \in(a, b)$, or the reverse inequalities hold with the same restrictions on $h$ and $k$. The inequalities ( 9 ) and (10) bear the same relation to the Lagrange Theorem as the inequalities ( 8 ) and (7) bear to Flett's Theorem.

REMARK 4. Assuming that both $f$ and $g$ are differentiable, one obtains, from (3) and ( 4 ), by dividing by $h$ and $k$, respectively, and then passing to the limit as $h \rightarrow 0+$ and $k \rightarrow 0+$, that

$$
\begin{aligned}
& f^{\prime}(c)[g(c)-g(a)] \leq g^{\prime}(c)[f(c)-f(a)], \\
& g^{\prime}(c)[f(c)-f(a)] \leq f^{\prime}(c)[g(c)-g(a)],
\end{aligned}
$$

which implies

$$
g^{\prime}(c)[f(c)-f(a)]=f^{\prime}(c)[g(c)-g(a)] .
$$

(One, of course, arrives at this conclusion, too, if inequalities reverse to (3) and (4) hold.) This is precisely the conclusion of Theorem 2 in [9], except that there $c$ could conceivably be $b$, which is excluded here. It should also be mentioned that the hypothesis

$$
\left[\frac{f^{\prime}(a)}{g^{\prime}(a)}-\frac{f(b)-f(a)}{g(b)-g(a)}\right]\left\{[g(b)-g(a)] f^{\prime}(b)-[f(b)-f(a)] g^{\prime}(b)\right\} \geq 0
$$

in Theorem 2 of [9] can be shown to imply hypothesis (iii) in the present Theorem 1 , except in the trivial case, when $[g(b)-g(a)] f^{\prime}(b)=[f(b)-f(a)] g^{\prime}(b)$. If $g^{\prime}$ never vanishes, then (1I) 
can be written in the form

$$
\frac{f(c)-f(a)}{g(c)-g(a)}=\frac{f^{\prime}(c)}{g^{\prime}(c)}
$$

and this explains why a theorem of this sort is called a "fractional mean value theorem". As soon as a fractional mean value theorem is established, one can prove Taylor like theorems with various forms of the remainder (for example, Lagrange's, Cauchy's or Schlömilch's form). See [9], [4], [5]. This order of ideas will not be pursued further here.

REMARK 5. Assuming that only $g$ is differentiable, one obtains from Theorem 1 (similarly as in Remark 2), that either

$$
D^{+} f(c) \leq \frac{f(c)-f(a)}{g(c)-g(a)} g^{\prime}(c) \leq D_{-} f(c),
$$

or

$$
D^{-} f(c) \leq \frac{f(c)-f(a)}{g(c)-g(a)} g^{\prime}(c) \leq D_{+} f(c) \text {. }
$$

This is the conclusion of Theorem 2 in [8], except that in [8], c could be conceivably $b$, which is excluded here. Hypothesis (12) appears also in Theorem 2 of [8], and hence, as pointed out in Remark 4, the hypothesis of the present Theorem $l$ is actually weaker, except in the trivial case, when $[g(b)-g(a)] f^{\prime}(b)=[f(b)-f(a)] g^{\prime}(b)$.

REMARK 6. The usual Cauchy fractional mean value theorem reads: If $f$ and $g$ are real valued functions continuous on $[a, b]$, differentiable on $(a, b)$, then there exists a number $c$ such that $[f(b)-f(a)] g^{\prime}(c)=[g(b)-g(a)] f^{\prime}(c)$. A generalization of the Cauchy Theorem, concerning Dini derivates, appears in the work of W.H. and Grace Chisholm Young [10, pp. 19-24]; roughly speaking, this generalization is related to Cauchy's Theorem in a similar way as inequalities (14) and (15) are related to equation (13). A further generalization of Cauchy's Theorem can be given as follows [4, Remark 4]: If $f$ and $g$ are real valued continuous functions on $[a, b]$, then there exists a number $c$ such that, either

$$
\begin{aligned}
& {[f(b)-f(a)][g(c)-g(c-h)] \leq[g(b)-g(a)][f(c)-f(c-h)],} \\
& {[g(b)-g(a)][f(c+k)-f(c)] \leq[f(b)-f(a)][g(c+k)-g(c)]}
\end{aligned}
$$

hold for all positive $h$ and $k$ such that $a-h \in[a, b], c+k \in[a, b]$, 
or both inequalities (16) are valid with inequality sign reversed, with the same restriction on $h$ and $k$. The conclusion of this generalized mean value theorem bears the same relation to the conclusion of the Cauchy mean value theorem as the conclusion of the Theorem 1 of this paper bears to the conclusion of Theorem 2 of [9] [roughly speaking, to equation (11)], except that, in the generalization to Cauchy's theorem, the numbers $h$ and $k$. are only restricted by inequalities $0<h \leq c-a, 0<k \leq b-c$.

\section{Partial converse}

In considering the possibility of a converse of Theorem 1 , only the case when $g(x) \equiv x$ will be taken into account. A natural converse of Theorem 1 would state that, if $f$ is continuous, and there exists a number $c$, with $a<c<b$, and positive numbers $\delta_{1}, \delta_{2}$ such that either

$$
\frac{f(c+k)-f(c)}{k} \leq \frac{f(c)-f(a)}{c-a} \leq \frac{f(c)-f(c-h)}{h}
$$

hold for $0<h \leq \delta_{1}$, and $0<k \leq \delta_{2}$, or the reverse inequalities are valid with the same restrictions on $h$ and $k$, then the graph of $f$ intersects its chord. However, this proposition is not true, as examples in Figure 1 and Figure 2 show. Nevertheless, the following theorem holds.

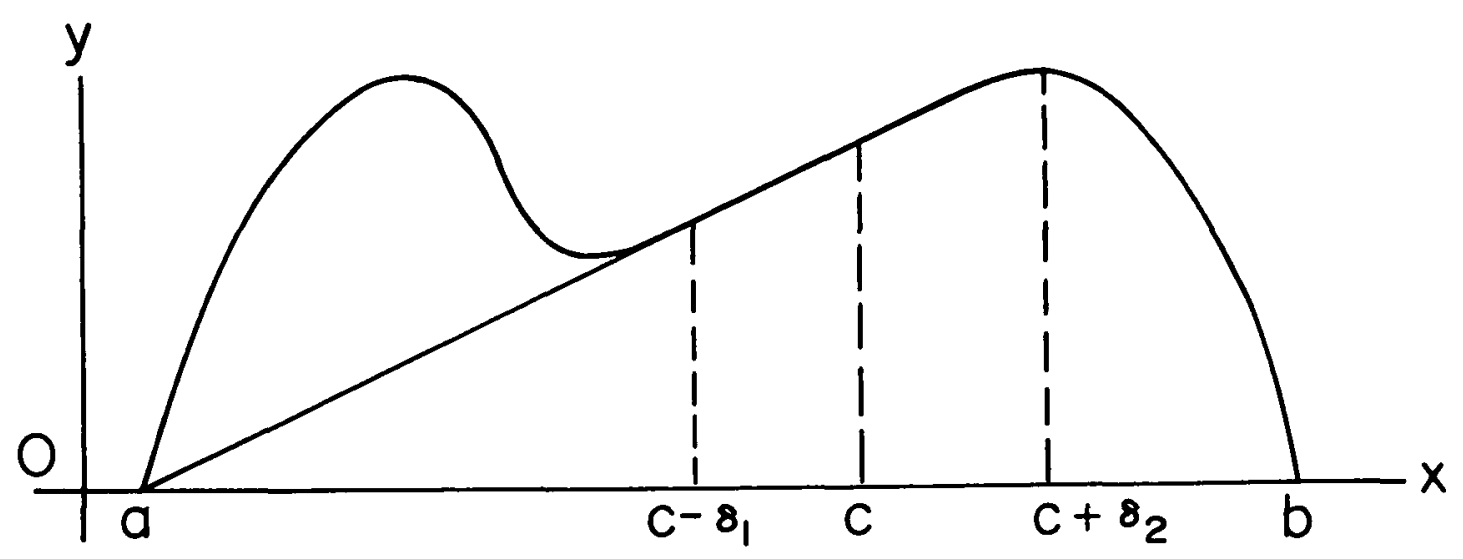

Figure 1 


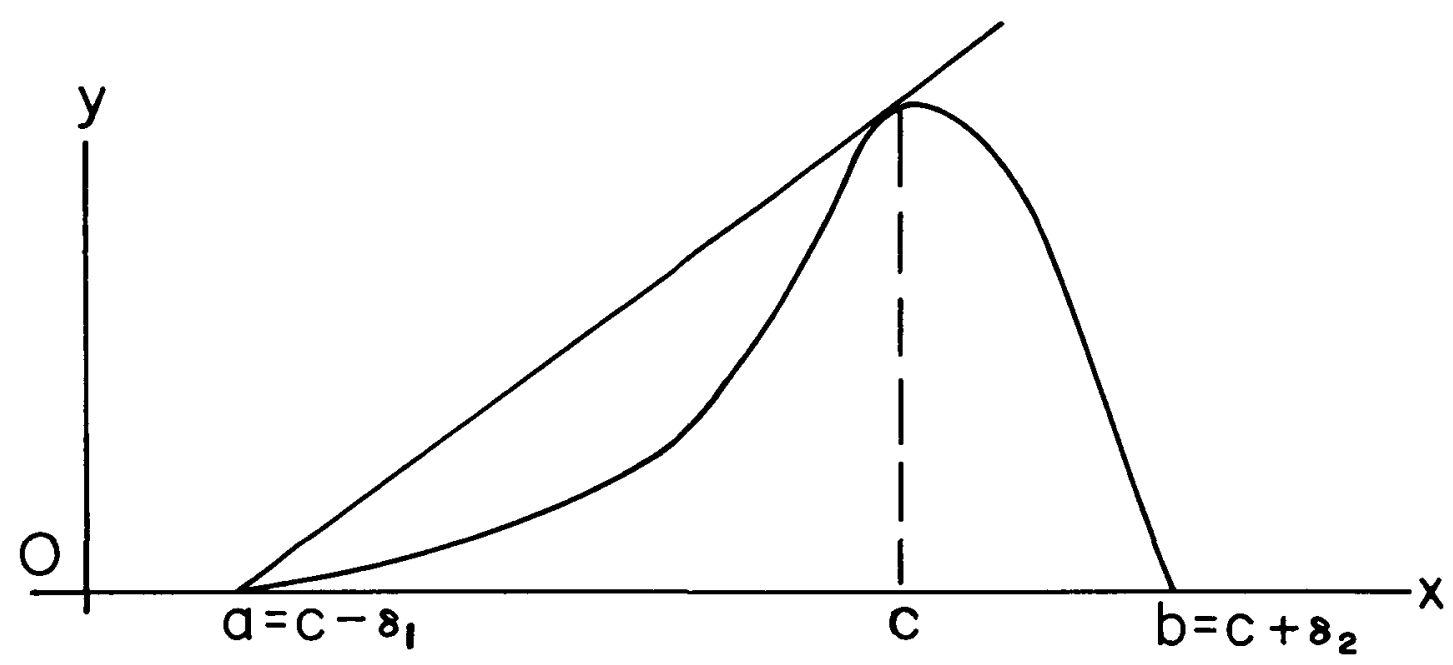

Figure 2

THEOREM 2. Let $f$ be a real valued continuous function on $[a, b]$. If there is a number $c \in(a, b)$ such that either the inequalities (17), or the reverse inequalities, hold for all positive $h$ and $k$, with $a \leq c-h, c+k \leq b$, then, either $f$ is linear on $[a, c]$, or there is $a$ number $d \in(c, b]$ and a number $\bar{x}$, with $a<\bar{x}<d$, such that

$$
\frac{f(\bar{x})-f(a)}{\bar{x}-a}=\frac{f(d)-f(a)}{\bar{d}-a} \text {. }
$$

(Thus, if (18) holds, then the groph of $f$ intersects its chord (intermally) between the points $(a, f(a))$ and $(d, f(d))$.

Proof. Suppose, first, that (17) holds. Then

$$
\frac{f(x)-f(a)}{x-a} \leq \frac{f(c)-f(a)}{c-a}
$$

holds, for $a<x \leq b$. Two cases arise. In the first case, the equality sign holds for all $x$, with $a<x<c$. In this case, $f$ is linear on $[a, c]$. In the second case, there is a number $x_{0}$, with $a<x_{0}<c$, such that strict inequality holds, in (19), for $x=x_{0}$. There are two subcases; either, 


$$
\frac{f(b)-f(a)}{b-a} \geq \frac{f\left(x_{0}\right)-f(a)}{x_{0}-a}
$$

or

$$
\frac{f(b)-f(a)}{b-a}<\frac{f\left(x_{0}\right)-f(a)}{x_{0}-a}
$$

If (20) holds, then the continuous function $H$, defined by

$$
H(x)=\frac{f(x)-f(a)}{x-a}
$$

has a value less than or equal to $\frac{f(b)-f(a)}{b-a}$ at $x=x_{0}$, by (20), and has, in view of (19), with $x=b$, a value greater than or equal to $\frac{f(b)-f(a)}{b-a}$ at $x=c$. Therefore, there exists a number $\bar{x} \in\left[x_{0}, c\right]$ such that

$$
\frac{f(\bar{x})-f(a)}{\bar{x}-a}=\frac{f(b)-f(a)}{b-a},
$$

that is, the equation (18) is satisfied for $d=b$. If (2l) holds, then the continuous function $H$ has a value less than $\frac{f\left(x_{0}\right)-f(a)}{x_{0}-a}$ at $x=b$, by (21), and has a value greater than $\frac{f\left(x_{0}\right)-f(a)}{x_{0}-a}$ at $x=c$, in view of (19) with $x=x_{0}$. Therefore, there exists a number $d$, with $c<d<b$, such that

$$
\frac{f(d)-f(a)}{d-a}=\frac{f\left(x_{0}\right)-f(a)}{x_{0}-a},
$$

that is, equation (18) is satisfied, with $\bar{x}=x_{0}$. If the inequalities reverse to (17) hold, then (17) holds with $f$ replaced by $-f$, and, therefore, the desired conclusion follows in this case also.

\section{Vector valued functions}

THEOREM 3. Let the functions $F$ and $g$ satisfy the following conditions:

(i) the vector valued function $F$ is continuous on $[a, b]$, and its values are in a linear normed space $B$ (with the norm 
denoted by \|\|$)$; the real valued function $g$ is continuous on $[a, b]$,

(ii) $g(x)>g(a)$, for $a<x \leq b$,

(iii) either there exists a number $\bar{x}$ such that

$$
\left\|\frac{F(\bar{x})-F(a)}{g(\bar{x})-g(a)}\right\|=\left\|\frac{F(b)-F(a)}{g(b)-g(a)}\right\| \text {, }
$$

or, the limit $\lim _{x \rightarrow a^{+}}\left\|\frac{F(x)-F(a)}{g(x)-g(a)}\right\|$ exists, and

$$
\lim _{x \rightarrow a^{+}}\left\|\frac{F(x)-F(a)}{g(x)-g(a)}\right\|=\left\|\frac{F(b)-F(a)}{g(b)-g(a)}\right\| .
$$

Then, there exists a number $c \in(a, b)$ and a positive number $\delta$, such that, either

$$
\|F(c)-F(a)\|[g(c)-g(c-h)] \leq[g(c)-g(a)]\|F(c)-F(c-h)\|,
$$

for $0<h<\delta$, or

$$
\|F(c)-F(\alpha)\|[g(c+h)-g(c)] \leq[g(c)-g(a)]\|F(c+h)-F(c)\|,
$$

for $0<h<\delta$.

Proof. Using Theorem 1 for $f$, where $f(x)=\|F(x)-F(\alpha)\|$, one obtains: If inequalities (3) and (4) hold, then one obtains, from (3), that

(24) $\|F(c)-F(a)\|[g(c)-g(c-h)] \leq[g(c)-g(a)][\|F(c)-F(a)\|-\|F(c-h)-F(a)\|]$, for $0<h \leq \delta=\min \left(\delta_{1}, \delta_{2}\right)$, and, by the triangle inequality, that

$$
\|F(c)-F(a)\|-\|F(c-h)-F(a)\| \leq\|F(c)-F(c-h)\| \text {. }
$$

Inequality (22) now follows, using (ii) with $x=c$, from (24) and (25). If, on the other hand, the inequalities reverse to (3) and (4) hold, then one obtains, from the inequality reverse to (4), that

(26) $[\|F(c+h)-F(a)\|-\|F(c)-F(a)\|][g(c)-g(a)] \geq[g(c+h)-g(c)]\|F(c)-F(a)\|$, for $0<h \leq \delta=\min \left(\delta_{1}, \delta_{2}\right)$, and, by the triangle inequality, that

$$
\|F(c+h)-F(a)\|-\|F(c)-F(a)\| \leq\|F(c+h)-F(c)\| \text {. }
$$

Inequality (23) now follows, using (ii) with $x=c$, from (26) and (27).

THEOREM 4. Let the fronctions $F$ and $g$ satisfy conditions (i) and 
(iii) of Theorem 3, and let $g$ be strictly monotonic. Then, there exists a number $c \in(a, b)$ and a positive number $\delta$, such that either

$$
\left\|\frac{F(c)-F(a)}{g(c)-g(a)}\right\| \leq\left\|\frac{F(c+h)-F(c)}{g(c+h)-g(c)}\right\| \text {. }
$$

for $0<h \leq \delta$, or

$$
\left\|\frac{F(c)-F(a)}{g(c)-g(a)}\right\| \leq\left\|\frac{F(c)-F(c-h)}{g(c)-g(c-h)}\right\|
$$

for $0<h \leq \delta$.

If, further, $F$ is strongly differentiable on $(a, b)$, and $g$ is differentiable on $(a, b)$, then

$$
\left\|\frac{F(c)-E(a)}{g(c)-g(a)}\right\| \leq\left\|\frac{F^{\prime}(c)}{g^{\prime}(c)}\right\| .
$$

Proof. If $g$ is strictly increasing, then hypothesis (ii) of Theorem 3 holds, and (29) and (28) follow directly from (22) and (23), respectively. If $g$ is strictly decreasing, one considers $-g$, instead of $g$. If $F$ and $g$ are differentiable, then, passing to the limit, as $h \rightarrow 0+$, in either (28) or (29), one obtains (30).

REMARK 7. Theorem 4 is a sort of a "fractional Flett-Trahan mean value theorem for vector valued functions". Using the Hahn-Banach extension theorem, it is not difficult to extend Theorem 4 to the case when $F$ is only weakly differentiable (see, for example, [1]).

\section{References}

[1] A.K. Aziz and J.B. Diaz, "On a mean value theorem of the weak differential calculus of vector-valued functions", Contributions to Differential Equations 1 (1963), 271-273.

[2] Ralph P. Boas, Jr, A primer of real functions (Carus Mathematical Monographs, No. 13. Mathematical Association of America; John Wiley \& Sons, New York, 1960).

[3] J.B. Diaz and R. Výborný, "On mean value theorems for strongly continuous vector valued functions", Contributions to Differential Equations 3 (1964), 107-118. 
[4] Joaquin B. Diaz and R. Výborný, "A fractional mean value theorem, and a Taylor theorem, for strongly continuous vector valued functions", Czechos lovak Math. J. 15 (90) (1965), 299-304.

[5] Friedhelm Erwe, Differential and integral calculus (translated by 8. Fishel; Oliver \& Boyd, Edinburgh; Hafner, New York, 1967).

[6] T.M. Flett, "A mean value theorem", Math. Gaz. 42 (1958), 38-39.

[7] T.V. Lakshminarasimhan, "A mean value theorem - an extension", Amer. Math. Monthly 73 (1966), 862-863.

[8] Simeon Reich, "On mean value theorems", Amer. Math. Monthly 76 (1969), 70-73.

[9] Donald H. Trahan, "A new type of mean value theorem", Math. Mag. 39 $(1966), 264-268$.

[10] W.H. Young and Grace Chisholm Young, "On derivatives and the theorem of the mean", Quart. J. Pure App 2. Math. 40 (1909), 1-26.

\footnotetext{
Rensselaer Polytechnic Institute,

Troy,

New York, USA;

University of Queensland,

St Lucia, Queensland,

and

Rensselaer Polytechnic Institute,

Troy,

New York, USA.
} 